\title{
Proposing a method for estimating the real micro profile of a milling tool
}

\author{
Mihai Boca $^{1, *}$, Mihaita Horodinca ${ }^{2}$ and Marian-Octavian Andronic ${ }^{1}$ \\ ${ }^{1}$ Gheorghe Asachi Technical University of Iasi, Machine Manufacturing Department, Iasi, Romania \\ ${ }^{2}$ Gheorghe Asachi Technical University of Iasi, Machine Tools Department, Iasi, Romania
}

\begin{abstract}
Manufacturing Engineering domain generally uses for creating virtual work piece profiles or tool representations descriptive parameters or simplified drawings. More recent and accurate methods such as the present study uses optical devices for obtaining virtual projections of work pieces/tools. This paper presents an experimental method applied in order to obtain the profile for a given cutting tool, which can offer in addition possibility to estimate the cutting tool wear, without using such built-in machine-tool device. The experimental values obtained are materialized by the accuracy which characterizes the accurate profile of the measured cutting tool. This fact can make a difference between a classical measurement method applied outside of machine tool sector and a new one provided by a device which offers the solution to measure a work piece directly on the machine tool, without modifying the actual work piece orientation conditions and at a low price.
\end{abstract}

\section{Introduction}

In the Machine Manufacturing field, especially in the cutting processes sector, the necessity of increasing the productivity has generated the possibility to integrate into an MTDW system (Machine-Tool Device Work piece) different kinds of instruments/devices for measuring work piece constructive parameters or after processing. Other machine tools developers have introduced several instruments for monitored and make adjustment during processing. This fact was done in order to maintain the same conditions of processing for every manufactured work piece. As example, the Okuma processing centres [1] have the capability to monitories the machine-tool behaviour given by thermal phenomenon or chatter, through a series of some intelligent tools as Machining Navi, Thermo friendly concept and so on. Many of these improvements have conducted to significant results obtained after work piece processing.

On the other hand, the actual generation of intelligent machine-tools will need to become more complexes than now. Thus, they should monitors more parameters than now. This idea, based on the result collected after several measurements made directly on the machine, will allow having more accurate active control under the process. This fact may include various mechanisms for measuring several parameters such as: stiffness, roughness,

\footnotetext{
*Corresponding author: mihaitzaboca@yahoo.com
} 
roundness and others, during processing. These parameters will serve to make active or offline adjustments. Of course, these measurements will be made without contact with the processed work piece, as it will show in this paper.

In our proposed study an inspection instrument to study the shape of a cutting tool was used. In this way, it was proposed an offline method for measuring the profile of a milling tool. This method will suppose that at the end of the cutting program, the cutting tool will be subject to a measurement. This fact will provide necessary information's in order to make a graphical representation of this cutting tool and will offer the possibility of measurement different kinds of cutting tool profile as: wear status, radius modifications, and so on. Besides this, the presence of this device around the machine tool will offer the possibility to integrate such measurement system on the machining centre and to use it into an automated process of data measurement.

\section{General considerations}

In the previous studies made until now $[2,3,4,5]$ it was highlighted the possibility to measure the work pieces processed on a lathe $[2,3]$, especially on measuring the radial deviation of a shaft and to represent it into a virtual environment. The technical literature also presents some aspects concerning a study which presents the possibility to measure a work piece, using an optical sensor, from a milling machine [3] in order to measure the geometry of the processed part. The author highlighted the opportunity offered by the present of this integrated device into the machine structure as a possibility to extend the range of different measurement applications on this machine. The next step of this study it should become the integration of the measurement procedure on the machine-tool controller. Other study [6] presents also a measurement method applied to determine the roundness of a work piece, during the processing, with non-contact probe. Such measurement study supposed to determine the roundness error for every work piece processed by turning. A similar measurement device is presented in the reference [7] where a Form Measuring Machine (FMM) is used for made different geometric measurements. In general, the problem of different kinds of errors measurement it was intensively treated, but not in a way to rebuild a particular work piece / cutting tool.

Thus, in all cases, an experimental stand which can be used to measure directly on the machine-tool several geometric dimensions, at a low price due to its individual components, appears as a necessity.

\section{Methodology and procedural aspects}

A high importance in this study is granted to measure with accuracy all the geometrical and dimensional characteristics of the considered milling tool in order to make an accurate graphical representation of it.

The device build and used in this study was used also for measuring the roundness and the radial deviation of a work piece processed by turning. From this reason, the measuring stage will analysed the shape of the cutting edges.

In this way, a measurement's stand with possibility to use the experimental stand on other different machine tools it was achieved. 


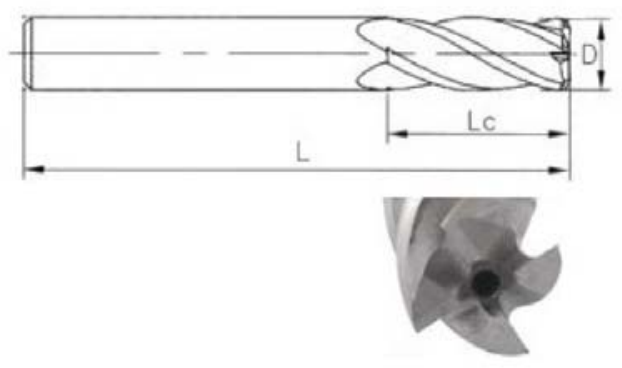

Fig. 1. Shape and dimensions of the considered milling tool.

The subject of the measure is represented by a milling tool, graphically represented in the figure 1, with four teeth placed helical on the considered cylindrical shape with a diameter of $16 \mathrm{~mm}$. In the Figure 1 with $\mathrm{D}$ was represented the milling tool diameter with the size of $16 \mathrm{~mm}$, with Lc the active cutting length with the size of $45 \mathrm{~mm}$ and with $\mathrm{L}$ the total length with the size of $95 \mathrm{~mm}$ (according to [2]). The tool profile was obtained by mounting the milling tool into the tail stock of a considered lathe (Figure 2) in order to perform a such measurement and to verify the data obtained.

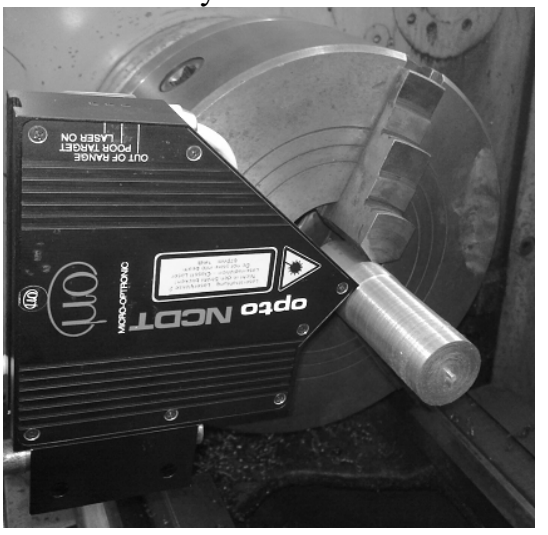

Fig. 2. Working position for the optical sensor and work piece used for measurements.

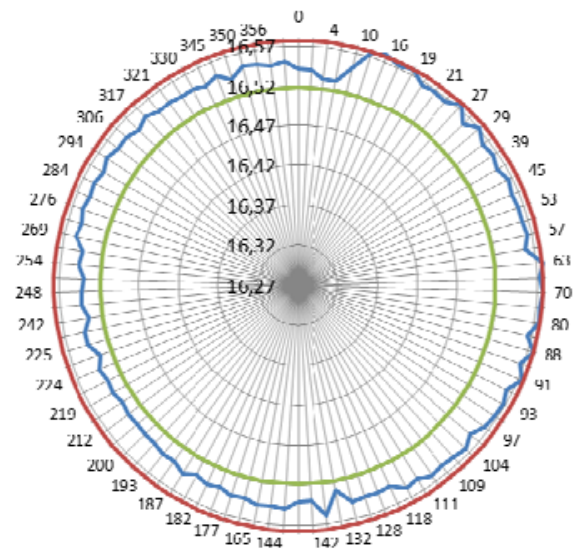

Fig. 3. Graphical representation of the frontal section of the test work piece.

First of all, a scanning test was performed with the experimental stand in order to verify the measurement accuracy. Thus, after the first measurement performed on the test work 
piece it was obtained a graphical representation such as the one from the Figure 3. In this figure it is observed that the obtained profile is varying between 16.57 and $16.52 \mathrm{~mm}$ that means the measured profile is fitting between the prescribed tolerances and have a deviation of $0.05 \mathrm{~mm}$. In this figure with 4 is represented the optical device 1 used for representing the profile of the milling tool, with 2 the considered work piece for the test accuracy and with OMR the optical measurement range. The OMR length was imposed by the measurement rage available for the optical device selected, the optimum distance being established manually with help of tree LEDs place on the optical device body (see Figure 2 ). The measurement test for the milling tool was performed on similar conditions as it was obtained the shape and dimensions of the considered test work piece.

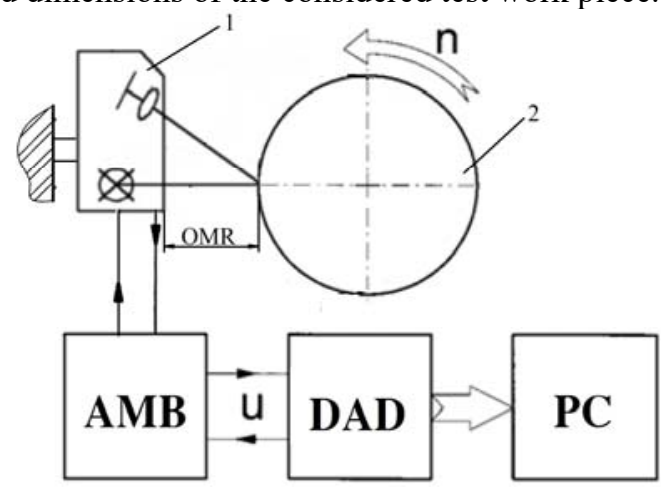

Fig. 4. The scanning test performed with the experimental stand.

Using the tail stock round movement and the possibility to move the optical sensor mounted on the transversely carriage on the lathe along $\mathrm{x}$ axis, it was possible to establish the transversely profile of the measured tool.

For measuring the geometric and dimensional tool profile, an optical sensor (1) type ILD 2000-20 (Micro-Epsilon, Germany) was used (Figure 4). The measurement principle consists into sending a laser beam on the tool surface in order to be reflected and received by the optical sensor. It has to be mentioned that the optical device is based on laser triangulation principle. The signal received gives information about the measured shape and also, about the positions of the various points considered along the longitudinal surface of the tool. The signal received was processed using a Pico scope ADC 212-50, Pico technology U.K, transformed trough a measurement bridge Acquisition Data's (DAD), type N2302, produced by IEMI company, an Aquision and Measurement Bridge (AMB) and graphically represented on a personal computer (PC). The $\mathrm{u}$ letter represents the signal transmitted between experimental stand components. All sorted and processed data were arranged based on a couple of numerical filters applied in MatLab software.

The tool evaluation it was made on the radial profile of this, as can be seen on the Figure 4. The profile described was established by rotation of the tool with a speed of $532.7 \mathrm{rot} / \mathrm{min}$, this measured value being determined through measurement, according to the study [2].

\section{Experimental results and discussions}

Figure 5 presents a graphic detail over the time evolution of the signal provided by the optical sensor and transformed into relative movement. The period of time for this representation is exactly one second. The obtained curve was established by performing several measurement stages on the measured tool profile, also to establish the accuracy of measurements. 
On the Figure 5 can be noted that each alternation of the measured signal corresponds to a tooth passing of the laser beam. Thus, the negative picks are associated with the tool teeth $\mathrm{T} 1, \mathrm{~T} 2, \mathrm{~T} 3$, and T4. The term $\mathrm{P}$ is associated with the reference period of time, mentioned above, in which was performed a complete rotation of the tool.

Furthermore, the graphical representation from the Figure 5 can be transposed on polar coordinates considered on a reference length, aspects highlighted on the Figure 6.

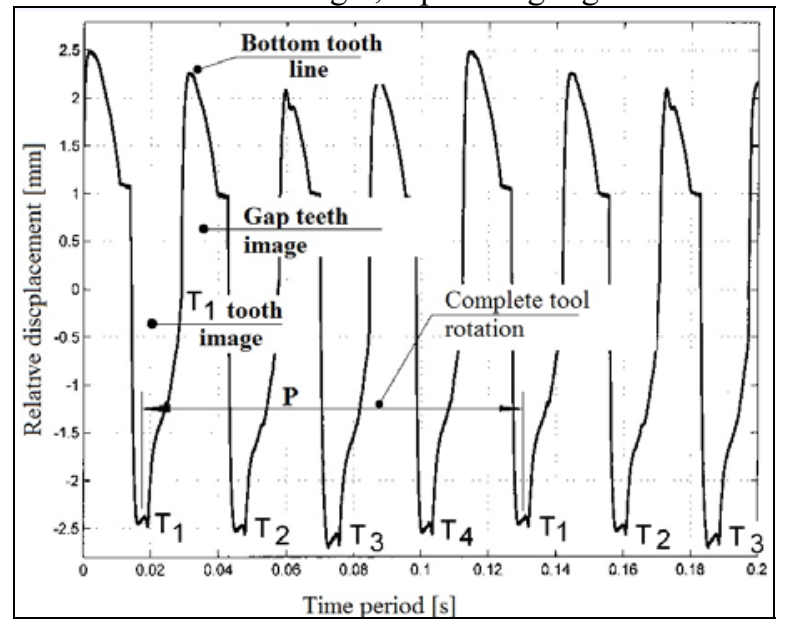

Fig. 5. The rectangular signal representation of the considered tool profile.

Thus, in this representation the profile of the tool was rotated with an angle of 360 degree. The reference length was considered equal with 1/8.8794 seconds, period which corresponds to constant sequence multiplied from the measured signal. Thus, this length marked on the representation with $a$ symbol was assumed with the succession rotation of the four tool teeth. According to the previous remarks, the Figure 6 presents the radial profile of the measured tool. Also, Figure 6 reveal the diameter of the measured tool placed on the circle with the radius of $8 \mathrm{~mm}$. Of course, the representation is assumed as radial tool profile in the measured section, the last plane section of the tool.

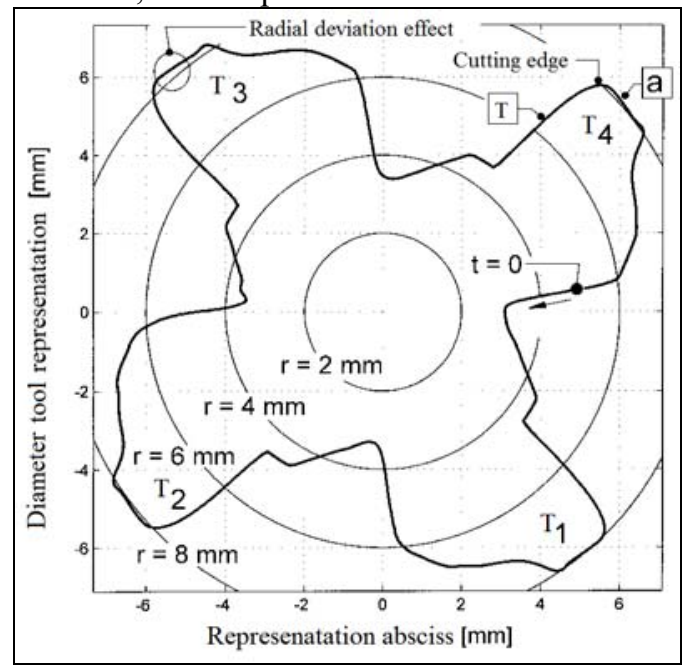

Fig. 6. The radial tool profile of the milling tool. 
The obtained result gives a perspective view under the measured tool profile. In this situation, the last representation allows to make references between the real tool profile and the graphical representation of it, regarding the shape and its dimensions.

It can be mentioned that the tool profile description is made point with point. This fact is done due to the presence of some deviations from the normal position of the laser beam on the measured surface, variations which can influence negatively the obtained result. Also, a significant influence is manifested by the mediation effect applied under the measured signal, which can also influence the obtained result.

As main advantage related to the Figure 6 is represented by the possibility to obtain a $3 \mathrm{D}$ profile for a supposed work piece or a supposed tool, in the manner in which was presented in this study.

In this present study, the number of describing movements which characterize the considered object is increased with a controlled movement along the longitudinal machine axis. Other advantage, after the result obtained, recommends the implementation of the presented method in order to make an active control of a work piece/tool directly on the machine tool as lathes, or milling machines, where a rotation movement is present.

\section{Conclusions}

This study presents the possibility to use an optical sensor as an instrument to appreciate the geometry of a milling tool and give ideas about dimensions of it. The measurement can be performed using in addition a third movement along the measured object. The experimental stand can be placed at any machine tool which performs cutting processes and the cutting tool or work piece is turning.

The presented procedure and the experimental results highlight the main advantage of the proposed stand, in order to integrate it into machine tool structure. In every moment, with the data obtained, the state of manufacturing process can be known. In this way, the machine tool operator can use this stand as an active system adapted for making in time adjustments, applying tool corrections, all these in order to avoid any kinds of errors which can occur during processing.

\section{References}

1. Okuma Intell. Tech., available at https://www.okuma.com/intelligent-technology, accesed at 15.03.2018

2. $16 \mathrm{~mm}$ End Mill, available at https://www.sandvik.coromant.com, accesed at 15.03.2018

3. S. Mekid, K. Vacharanukul, Measure. 44762 (2011)

4. M. Boca, G. Nagît, M. Horodinca, F. Negoescu, A.-M. Mihalache and M.-I. Ripanu, App. Mech. and Mat. 657 (2014)

5. M. Boca, G. Nagit, M. Horodinca, App. Mech. and Mat. 809 (2015)

6. N. Meier, A. Georgiadis, AMA Conf. 5126 (2013)

7. O.V. Zakharova, I.N. Bobrovskijb, A.V. Kochetkovc, 150 ( 2016 )

8. M. Sienilo, Adv. in Intell. Systems and Comp. 393 (2016) 